American Journal of Applied Sciences 5 (5): 553-558, 2008

ISSN 1546-9239

(C) 2008 Science Publications

\title{
Numerically Studying the Effect of Stiffening Rings on Reducing the Thickness of Torospherical Heads under Pressure on Their Concave Side
}

\author{
Sa'id Golabi, Ali Ghorbanpour-Arani and Arash Zamani \\ Department of Mechanical Engineering, University of Kashan, Kashan, Iran
}

\begin{abstract}
The high thickness of heads used in pressure vessels is always one of the main concerns of designers and manufacturers. A thorough study has been conducted on all types of heads including torospherical heads, with external and internal pressure to reduce their thickness using stiffening rings by finite element technique. Here the result of analysis on heads with pressure on their concave side is presented. The results include a method for determining the dimensions of the most suitable ring and its location on heads from one hand and its effect on reduction of the head thickness from the other hand. The result of analysis showed that using suitable stiffening ring may reduce up to $30 \%$ of the head thickness.
\end{abstract}

Key words: Pressure vessel, torospherical head, stiffening ring, finite element, strain gage

\section{INTRODUCTION}

Stiffening rings have mainly been one of the best solutions for reducing the thickness of cylindrical shells under external pressure. The codes and standards for effective cross section of these rings and their permissible joining procedure are completely explained in paragraph UG29 and UG30 of Division 1 and AD330 of Division 2, Section VIII, ASME codes ${ }^{[1,2]}$. The main function of the rings on cylindrical shells is to stop buckling of the shells subjected to external pressure based on the studies conducted by Donnel ${ }^{[3,4]}$. The more the number of the suitable rings, the less the shell thickness required to resist external pressure. However, to minimize the total weight of the shell the optimum number of rings can easily be found using a computer program ${ }^{[5]}$.

The effect of axial loads, external pressure and thermal loads on stiffened cylindrical shells has also been investigated in the past ${ }^{[4-8]}$. Theoretical and numerical calculation of stress in stress concentration points on both torospherical and ellipsoidal heads have also been investigated in the previous works ${ }^{[9-11]}$ and a complete method for using reinforcement plates for these points have been addressed in ASME codes.

On the other hand manufacturing torospherical and ellipsoidal heads can be divided into three most common methods. The small size heads are mainly formed using dies and hydraulic presses. The medium size heads that cannot be formed by dies in one stroke are designed with 2 radiuses, i.e., the crown and the knuckle radiuses. The crown radius is formed using spherical dies starting from the center of the head and the knuckle radius is then formed with flanging machines. The large thick heads are all made in several pieces by dies and then welded together. The main concern of this research is to come up with an approach to reduce the thickness of heads, use less material, smaller size forming machines and reducing total production costs. Reduction of even $1 \mathrm{~mm}$ in the thickness of a head has sometimes a great impact on the total costs of a head. For example when a designer comes up with a $26 \mathrm{~mm}$ plate for a head, a $30 \mathrm{~mm}$ plate shall be ordered since $26 \mathrm{~mm}$ plate might not be found. Using a stiffening ring may permit using a $25 \mathrm{~mm}$ thickness plate instead of a $30 \mathrm{~mm}$ one.

Since nothing has been mentioned in codes and standards about the application of stiffening rings or ribs for heads, current study has been planned with the following objectives: (a) study the feasibility of reducing the thickness of heads using stiffening rings and ribs; (b) determine the relation between the external or internal pressure, head dimensions and the location of the ring; (c) investigate the effect of ring cross section on reducing the thickness of head. Investigation was conducted on hundreds of stiffened and nonstiffened torospherical heads using COSMOS EXPRESS DESIGN 2004 finite element software. The effect of stiffening rings with various latitudinal diameters and certain cross section on maximum vonMisses (vM) stress was determined for each pressurized head and the location that leads to minimum maximum

Corresponding Author: Department of Mechanical Engineering, University of Kashan, Kashan 87317-51167, Iran Tel: + 98- 361- 5555333 Fax: +98-361-5559930 
von-Misses stress is proposed for attachment of a ring. The width and height of this ring is then altered to achieve minimum von-Misses (vM) stress and volume of material. The thickness of the head is then so reduced that the maximum stress of stiffened head be equal to the non-stiffened one.

SA -516 Gr 70 is the material selected for the heads with joint efficiency of 1 and corrosion allowance of $0.125 \mathrm{in}$. The ring at the current stage of research is considered as an integrated part of the head () and the result of study on welded rings will soon be published.

\section{ANALYTICAL MODELS}

The analysis has been conducted on 4 different head diameters with 3 different thicknesses of each on ASME heads described in UG $32^{[1]}$. The permissible pressure of heads are found using formulas in UG $32^{[1]}$. Then the maximum vM stress of each head with and without stiffening ring in various locations of the head was determined by analyzing hundreds of models. All the results are referenced to angle " $\alpha$ " starting from the line passing from the center of the knuckle and tangent line (Fig. 1). Similar to theoretical formulas (Procedure 2-2 of Moss $^{[2]}$ ) all the results indicate that the maximum von-Misses stress locates in the knuckle radius of the head and contrary to theoretical formulas only in a small area, not the whole knuckle and therefore the effect of the ring is investigated in this area.

Determining the best location for ring for all the models a reasonable arbitrary cross section were considered in this stage. The proposed width and height at this stage was from 1.5 to 2 times the head thickness. The main objective is to find the location of the ring. (The dimensions of the ring are altered in the next stage.) The angle on which there is minimum maximum vM stress by attaching a ring was found for all models. The results indicate that the best location for the rings is from $35^{\circ}$ to $40^{\circ}$. In Fig. 2 the result of analysis on a stiffened 84 inches OD, 3/8 inch thickness head with 331 psi internal pressure vs. different locations of ring is shown. The minimum maximum vM stress is obtained by attaching a ring at $\alpha=38^{\circ}$. The results of other models are shown in Table $1^{[14]}$.

After determining the most suitable location for a square cross section ring, the next objective is to find the most suitable cross sections for the ring. Various sizes of square cross section were modeled and the result for one model is shown in Fig. 3 in which the horizontal axes show the height (width) of the ring and the vertical axes shows the maximum von-Misses stress found in the vicinity of that ring. (Fig. 4). The curve in Fig. 3 shows a decreasing trend and increasing the size

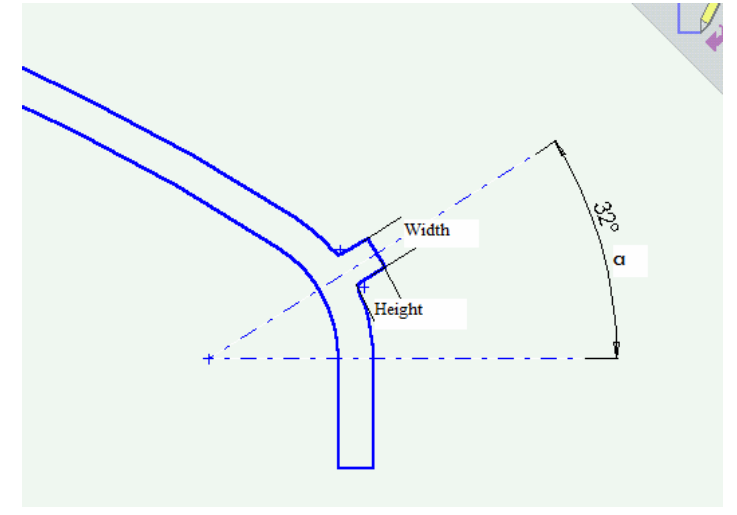

Fig. 1: Meridinal and latitudinal stresses experimental results versus the distance from the center of head with and without ring for $8 \mathrm{~mm}$ thickness head

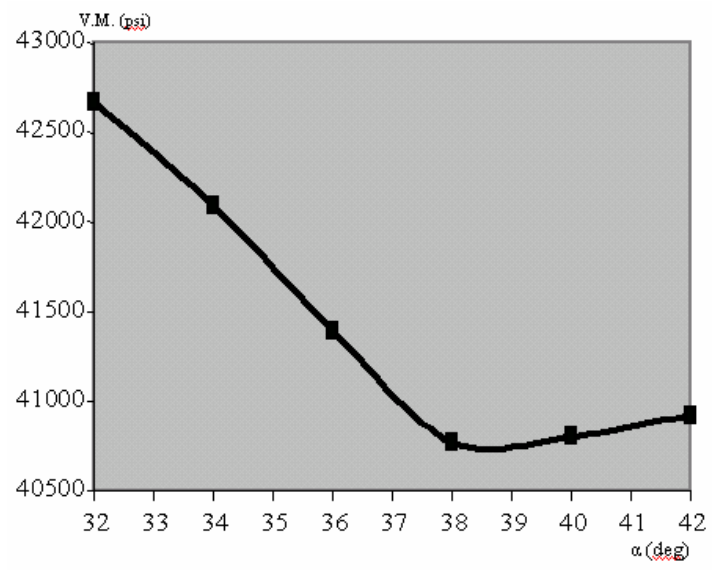

Fig. 2: Strain gauges arrangement

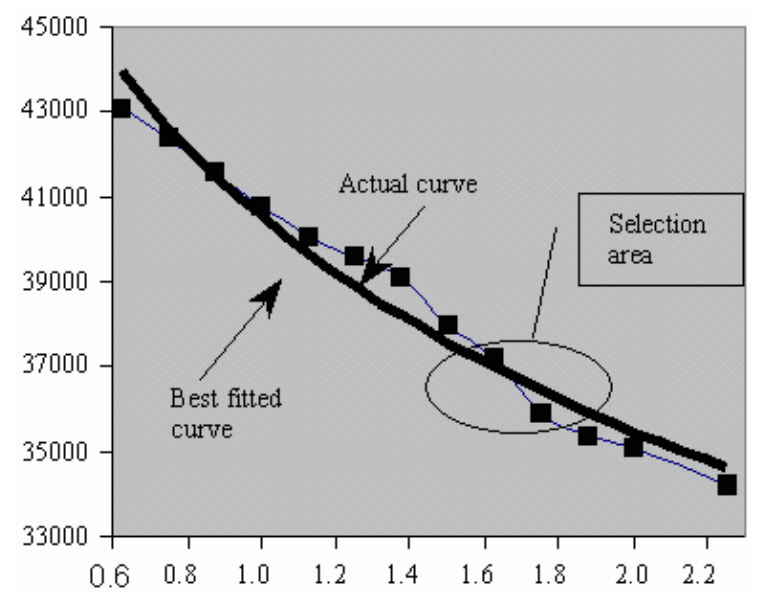

Fig. 3: Percentage of reduction in thickness vs. initial thickness 


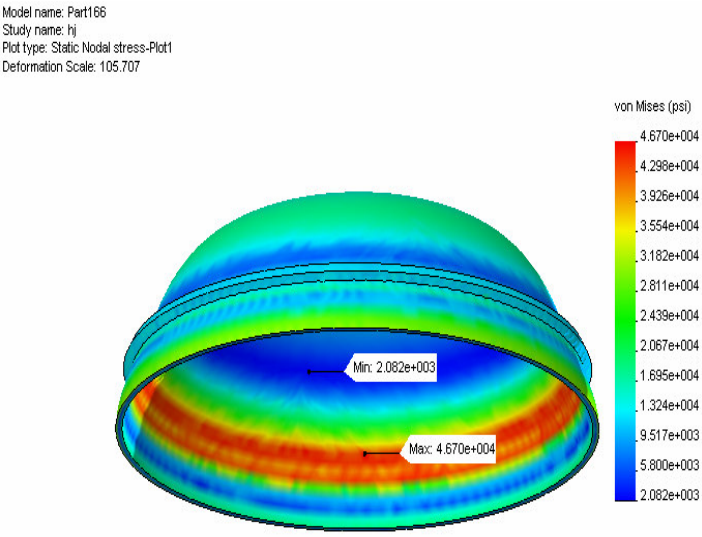

Fig. 4: Initial head thickness vs. final head thickness using stiffening ring

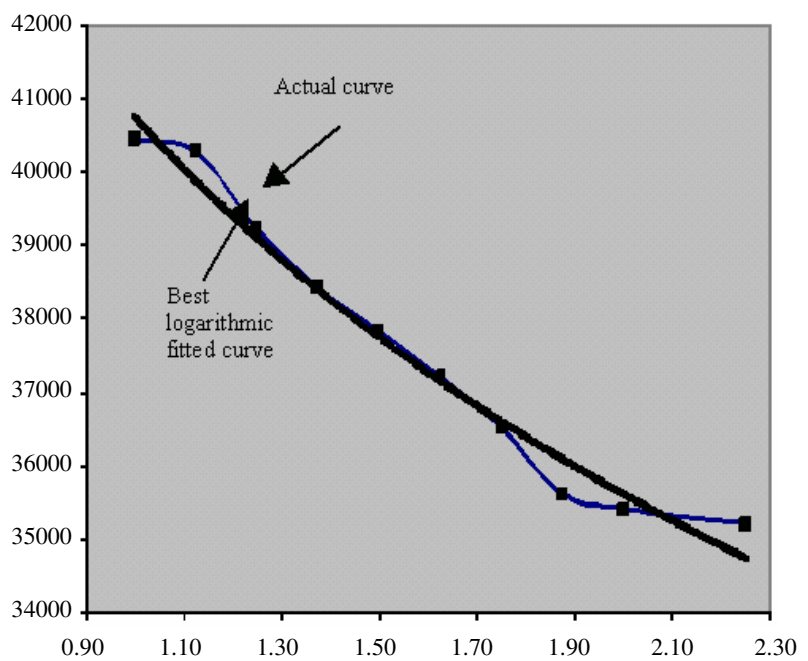

Fig. 5: Determining ring width from head diameter and thickness"

Table 1: Specifications of other heads

\begin{tabular}{lcc}
\hline OD (in) & Thickness (in) & Internal pressure (psi) \\
\hline 14 & $3 / 8$ & 476 \\
14 & $1 / 2$ & 770 \\
14 & $5 / 8$ & 1088 \\
48 & $3 / 4$ & 124 \\
\hline
\end{tabular}

of the ring will decrease the maximum stress. The curves of other models also show similar results.

Figure 3 and all other similar curves show that, the tangent to all curves becomes constant after a certain amount of ring cross section and there is not much decrease in the amount of maximum stress. Though the curve decreases constantly, it will be shown later that the total volume of the material will increase. Here the

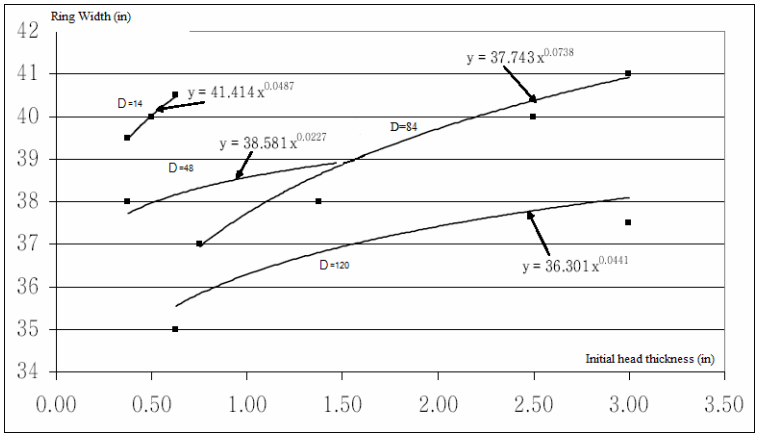

Fig. 6: Initial head thickness vs. final head thickness using stiffening ring

departing point between the curve and tangent to the curve where the tangent becomes constant was selected for the rings cross section as shown in Fig. 3 .

It was found that the stress in presence of small rings is even more than stress in heads without any ring because of stress concentration in the vicinity of the rings. On the other hand the larger rings do not much effect on decreasing the maximum stress and they also increase the weight and cost of manufacturing.

In fact 2 different approaches are involved in determining the cross section of the ring:

- Keep the width found in the previous section constant and determine the best height.

- Keep the height found in the previous section constant and search for the best width.

Both approaches were investigated and since the 2nd one shows better results, it is explained in here.

After determining the square cross section and the height of the ring using the method determined in the previous section, similar method was used to find the most suitable width for the ring. Figure 5 shows the result of analysis on the 84" model illustrated above and the results are shown on Fig. 2, 3 and 4. The actual curve clearly shows that there is not much reduction in the maximum stress after a certain amount of width. Similar to above section the width is selected from the zone that there is not much stress reduction.

Reducing the thickness: By determining the cross section of the ring in the steps explained above the vM stress is considerably reduced now. But before explaining the effect of changing the height instead of the width, the effect of the ring on reducing the thickness is described. The clue for reducing the 
Am. J. Applied Sci., 5 (5): 553-558, 2008

Table 2: The effect of ring on reducing the thickness

\begin{tabular}{|c|c|c|c|c|c|c|c|}
\hline $\begin{array}{l}\begin{array}{l}\text { Pressure } \\
(\mathrm{psi})\end{array} \\
\end{array}$ & $\begin{array}{l}\text { Initial head } \\
\text { thickness (in) }\end{array}$ & $\begin{array}{l}\text { External head } \\
\text { Dia. (in) }\end{array}$ & $\begin{array}{l}\text { Ring } \\
\text { height (in) }\end{array}$ & $\begin{array}{l}\text { Ring } \\
\text { width (in) }\end{array}$ & $\begin{array}{l}\text { Ring } \\
\text { location (deg) } \\
\end{array}$ & $\begin{array}{l}\mathrm{vM} \text { stress } \\
(\mathrm{psi})\end{array}$ & $\begin{array}{l}\text { Final head } \\
\text { Thickness (in) }\end{array}$ \\
\hline 331 & 1.625 & 84 & 1.625 & 1.625 & 38 & 37200 & 1.11 \\
\hline $\begin{array}{l}\text { Pressure } \\
(\mathrm{psi})\end{array}$ & $\begin{array}{l}\text { Initial head } \\
\text { thickness (in) }\end{array}$ & $\begin{array}{l}\text { External head } \\
\text { Dia. (in) }\end{array}$ & $\begin{array}{l}\text { Ring } \\
\text { height (in) }\end{array}$ & $\begin{array}{l}\text { Ring } \\
\text { width (in) }\end{array}$ & $\begin{array}{l}\text { Ring } \\
\text { location (deg) }\end{array}$ & $\begin{array}{l}\text { vM stress } \\
(\mathrm{psi})\end{array}$ & $\begin{array}{l}\text { Final head } \\
\text { Thickness (in) }\end{array}$ \\
\hline 140 & 0.5625 & 14 & $9 / 16$ & $5 / 16$ & 42 & 25010 & 0.475 \\
\hline 694 & 1.3750 & 48 & 1.25 & $10 / 16$ & 38 & 33300 & 1.352 \\
\hline 331 & 1.3750 & 84 & 1.5 & 1.625 & 38 & 36770 & 1.149 \\
\hline 589 & 3.0000 & 120 & 2.5 & 2.25 & 41 & 32260 & 2.818 \\
\hline
\end{tabular}

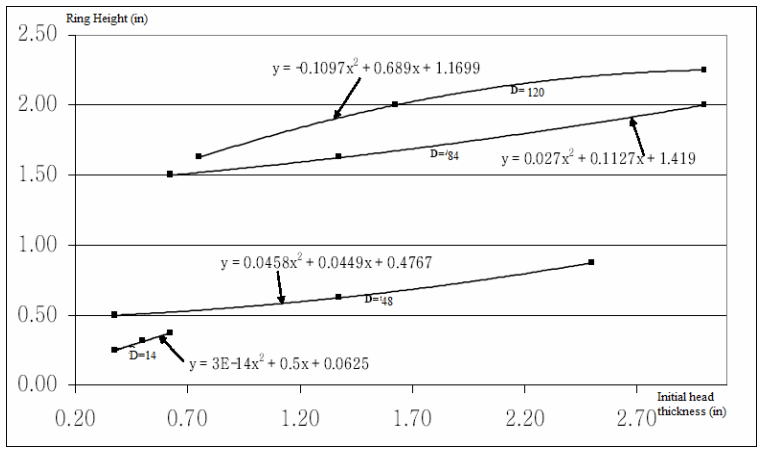

Fig. 7: Maximum vM (Psi) stress vs. the width of ring (in)

thickness is to find the thickness for a stiffened head whose ring dimensions found before so that its maximum von-Misses stress is equal to the nonstiffened one. The result of the analysis on some of the model explained within this study is abstracted in Table 2.

The analysis shows about $31.7 \%$ reduction in the thickness which is a considerable achievement. Not all other models show this much reduction in thickness, but all rings considerably effect the thickness of heads. The result of some other models is shown in Table 3.

As explained before, after determining the ring square cross section the most suitable width is found by keeping the height constant and varying the width.

One questions arises, i.e., why instead of the width, the height is not changed. This problem was investigated for all other models, ie, the curves for varying ring height was found but none gave the maximum vM stress less than the one found with varying width and consequently the head thickness found by varying the width was less than the one with varying the height. For example for the 84 inches head the initial head thickness was 3" and its thickness with a ring with varying width was reduced to 2.76 " while with varying height could maximum be reduced down to $2.82 "$. Similar results were found for all other

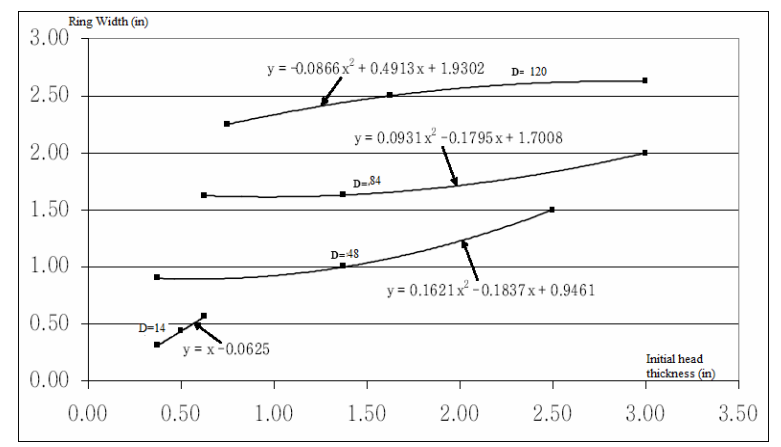

Fig. 8: Determining curve location from head diameter and thickness (in)

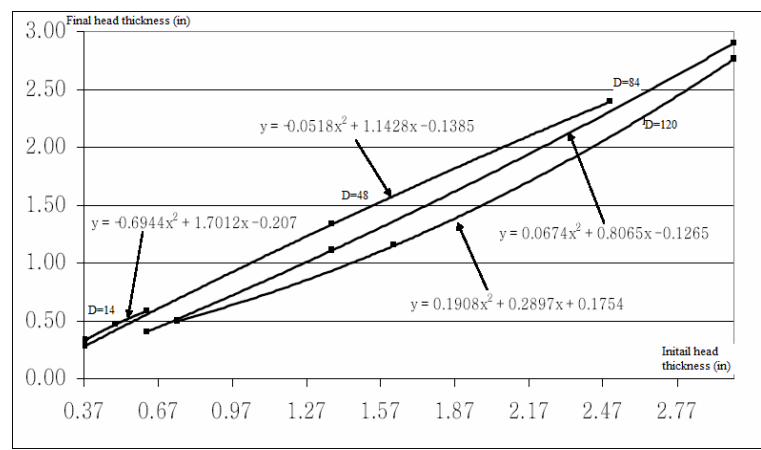

Fig. 9: Maximum vM (psi) stress of an 84 " head vs. the location of the ring

models. In most models the width of the models is interestingly found to be more than the height. In other words, despite stiffening rings used for cylindrical shells under external pressure, the axial moment of inertia of the ring as explained in $\mathrm{UG}^{2} 9^{[1]}$ has less effect in here. Apparently the thinner ring increases the stress concentration near the junction between the head and ring and more importantly distributes the longitudinal and latitudinal stresses in the maximum stress location, i.e., in the knuckle, more smoothly. Smoother stress flow can be achieved by considerably wider ring as it was found in the analysis. 


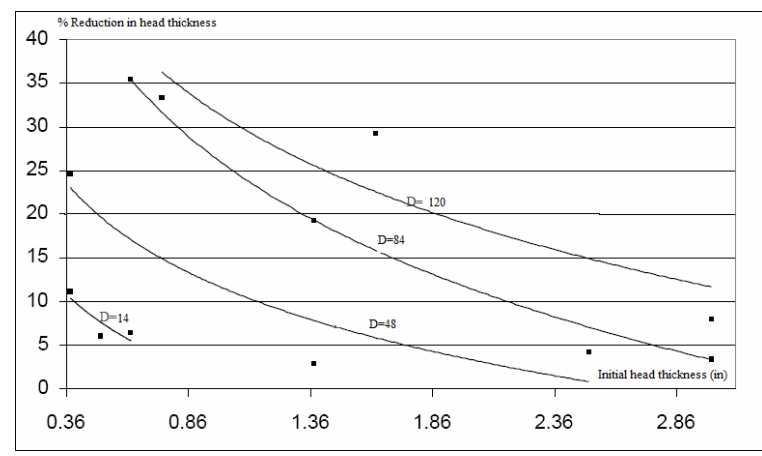

Fig. 10: A torospherical head with a ring at $\alpha=32^{\circ}$

Proposed instruction for choosing a ring: The tables explained above are the result of one by one finite element analysis of a large number of models. The main intention of this research is to find a general procedure for determining the location of ring, its size and its effect on reducing head thickness.

Since internal pressure on torospherical heads do not have considerable bending effect on the head thickness, the ring cross section axial moment of inertia $\mathrm{I}_{\mathrm{x}}$, (x axis is considered to be tangent to the head in the meridian direction passing from the center of ring cross section) was found not to be the main affecting parameter of the ring as cylindrical shells under external pressure explained in UG2 $9^{[1]}$. In contrary its effect is as the effect of rings on conical heads explained in appendix $1-5^{[1]}$.

Here the result of analysis on various types and sizes of heads are presented. Having the head diameter and thickness, one can easily find the best location for the ring using Fig. 7. From Fig. 8 the height and from Fig. 10 the width of the ring can be determined. Using aring with the height and width found from these figures the head final thickness can be found from Fig. 8 and the percentage of reduction in thickness can be determined from Fig. 10.

\section{CONCLUSION}

A new approach for reducing the thickness of torospherical heads with pressure on their concave side is presented in this paper. In this approach the most suitable stiffening ring with rectangular cross section for a head was so found that the maximum von-Misses stress of unstiffened head is equal to the stiffened head with reduced thickness.

The procedure finally addressed here consists of 4 steps:

- Determining the best location for the stiffening ring.

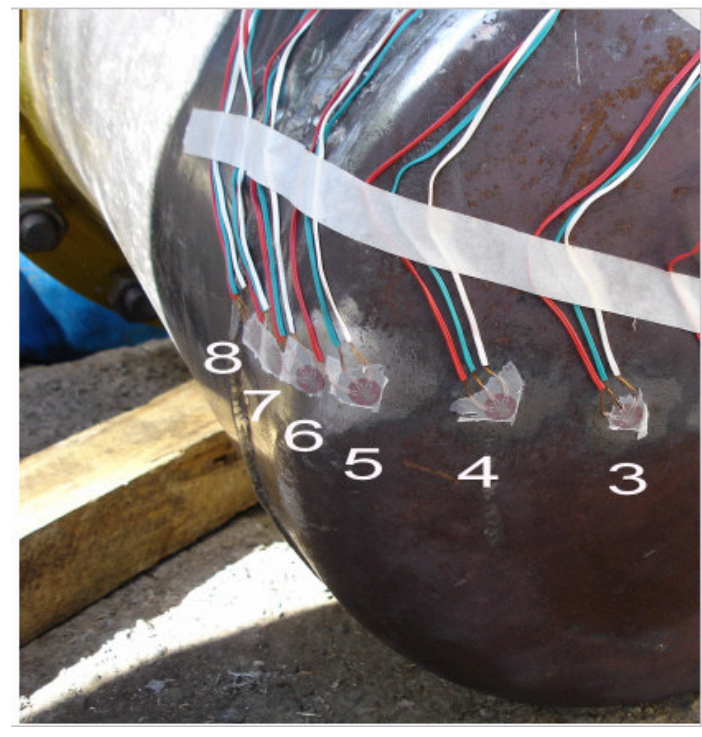

Fig. 11: Maximum vM stress (psi) vs. the size of square rings (in)

- Finding the most suitable height for the ring.

- Finding the most suitable width for the ring.

- Determining the stiffened head reduced thickness.

The analysis showed that using stiffening ring can in most cases be a successful technique for reducing head thicknesses and consequently the manufacturing costs. As can be seen from Fig. 10 It is especially more effective for larger and thinner heads and in some heads up to $30 \%$ thinner plate can be used. The results of finite element analysis are confirmed by experimental results. Heads with various ratio of diameter to thickness are tested using stain-gages. The experimental model with strain gages attached at specified locations are shown in Fig. 11 and the results of experiment and finite element analysis are presented in Fig. 12. The last figure shows the effect of stiffening ring on the reduction of maximum $\mathrm{vM}$ stress and a good conformance between finite element and experimental results. The experimental results will soon be published $^{[15]}$.

The rings are all interestingly should be welded on the knuckle radius of the head where no nozzle is allowed to attach. The result of investigation also indicated that not more than one ring can affect the maximum stress of the head and the maximum stress will remain around the ring and cannot be reduced more.

In consequence of above analysis the following researches have also been fulfilled and the results will soon be published: 

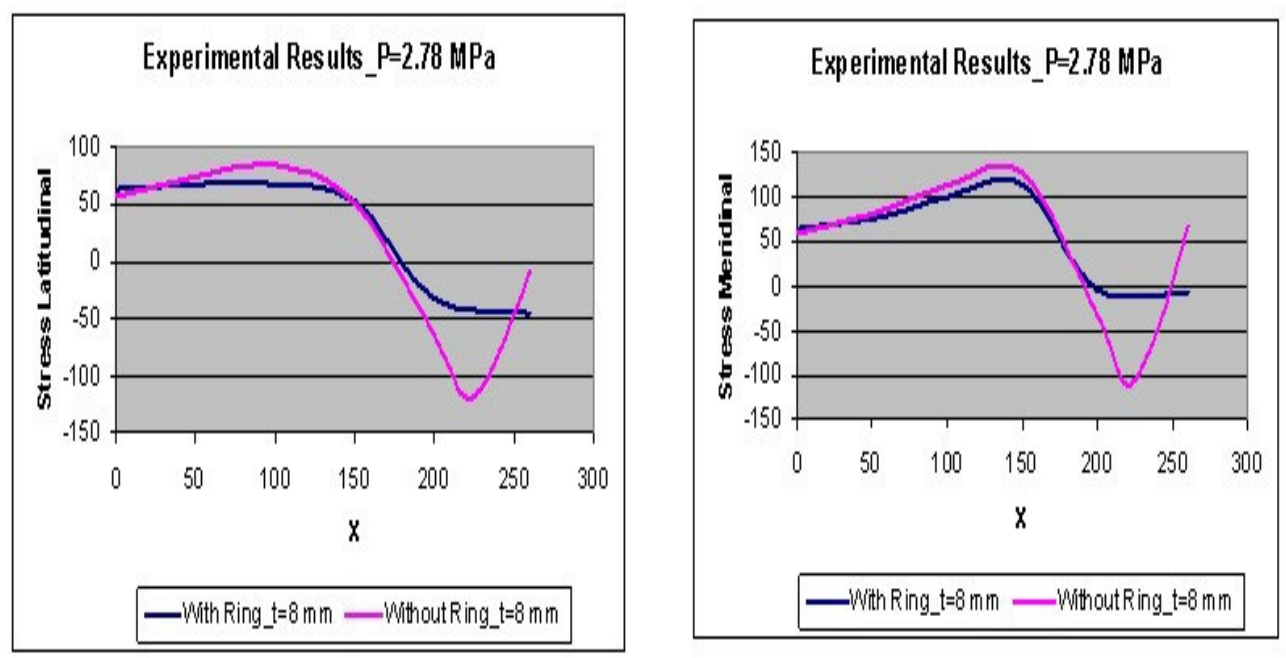

Fig. 12: The vM Stress of a stiffened head with pressure on its concave side

- The effect and size of rings on ellipsoidal and other types of heads.

- The effect and size of rings on heads with pressure on their convex sides.

- The result of analysis on welded rings in which the manufacturing aspects are also considered.

\section{REFERENCES}

1. American Society of Mechanical Engineers, 2004. Rules for Construction of Pressure Vessel. Division 1, ASME.

2. American Society of Mechanical Engineers, 2004. Rules for Construction of Pressure Vessel. Division 2, ASME

3. Donnel, L.H., 1950. Effect of imperfection on buckling of thin cylinders and columns under axial compression. Trans. ASME J. Applied Mech., 17: 73-83.

4. Donnel, L.H., 1956. Effect of imperfection on buckling of thin cylinders under external pressure. Trans. ASME J. Applied Mech., 23: 569-580

5. Giv, M. and M. Eskandari, 2003. Study and optimum design of cylindrical shells under external pressure. BSc Thesis, the University of Kashan, Faculty of Engineering.

6. Anderson, M.S., 1962. Combination of temperature and axial compression required for buckling of a ring stiffened cylinder. TN D-121, NASA

7. Chang, L.K. and M.F. Card, 1970. Thermal Buckling of stiffened cylindrical shells. Proceeding of the AIAA/ASME 11th structures, Structural Dynamics and Material conference, pp: 260-272.
8. Arbooz, J. and L.E. Sechler, 1976. On the buckling of stiffened imperfect cylindrical shells. AIAA J., 11: 1611-1617.

9. Li, J.Z., Y.H. Liu, Z.Z. Cen and B.Y. Xu, 1988. Finite element analysis for buckling of pressure vessels with ellipsoidal head. Int. J. Pressure Vessels Piping, 75: 115-120.

10. Magnucki, K. and J. Lewsinski, 2000. Fully stressed head of a pressure vessel. Thin-walled Structures, 38: 167-178.

11. Magnuckia, K., W. Szyca and J. Lewin'ski, 2002. Minimization of stress concentration factor in cylindrical pressure vessels with ellipsoidal heads. Int. J. Pressure Vessels Piping, 79: 841-846.

12. Moss, D.R., 2003. Pressure Vessel Design Manual. Gulf Publication Company, Houston, Texas

13. Hasandokht, S.K., 2004. Ring design and optimization of a torospherical head thickness with pressure on its concave side using finite element. BSc Thesis, the University of Kashan, Faculty of Engineering.

14. Zamani, A., 2004. Ring design and optimization of a torospherical head thickness with pressure on its convex side using finite element. BSc Thesis, The University of Kashan, Faculty of Engineering.

15. Tahvilian, A.M., 2006. The effect of stiffening rings on torospherical heads using finite element and experimental results. MSc. Thesis, Faculty of Engineering, the University of Kashan, Iran. 